\title{
A MAPREDUCE FRAMEWORK FOR DETECTION OF TANDEM REPEATS IN DNA SEQUENCES
}

\author{
${\text { Vandanababu } \mathrm{T}^{1} \text {, Raju Bhukya }}^{2}, \mathrm{D} \mathrm{Veeraiah}^{3}$ and P. Victer Paul ${ }^{3}$ \\ ${ }^{1}$ Department of Computer Engineering, Government Polytechnic Daman \\ ${ }^{2}$ Department of CSE National Institute of Technology Warangal \\ ${ }^{3}$ Department of Computer Science and Engineering, Vignan's Foundation for \\ Science, Technology \&Research, Guntur, Andhra Pradesh, India \\ vandana9925@gmail.com,raju@nitw.ac.in,d.veeraiah@gmail.com, \\ victerpaul@gmail.com
}

\begin{abstract}
DNA data produced by highly parallel next generation sequencing technologies entering into the field of bioinformatics as a flood, but traditional single processor based tandem repeat computing methods are not able to keep that pace. Microsatellite, also named as simple sequence repeats(SSR) with size 1-6 bp, are tandem repeats in DNA data is a sequence of two or more contiguous copies of same nucleotide sequence. These are playing very important roles in the fields of genetic diversity, forensic applications, genetic markers, mapping studies of human genome, and in disease diagnosis. There are many tools exist to detect these repeats, most of them were implemented around many assumed constraints, implemented on available hardware to minimise execution time; Existing tools possess different kind of advantages and disadvantages with each other. Hence a tool with exhaustive search for all tandem repeats is required. We proposed an efficient and constraint free Hadoop MapReduce based approach for detecting tandem repeats. It works in parallel and takes functional advantages of key -value based implantations. It displays one kind of repeat and all its occurrence in entire file a once, consolidates all kinds of repeats. Our experimental results are more readable, easily interpretable. It shows that the proposed approach more efficient with respect to increased data size when other tools have limitations.
\end{abstract}

Keywords - Next generation sequencing, Tandem repeats, Exhaustive search, Hadoop MapReduce framework, Map task, Reduce task

\section{INTRODUCTION}

\subsection{TANDEM REPEATS}

A large amount of our genome is comprised of tandemly repeated DNA. Microsatellites also known as short tandem repeats (STR) or simple sequence repeats (SSR) are tandemly repeated tracts of DNA composed of 1-6 base pair (bp) long units which are widely spread in both prokaryotic and eukaryotic genomes [1]. These are classified perfect (ACACACACACAC here motif is AC repeated 6 times), imperfect (ACACAAACACAC here motif is AC repeated 6 times, at third time instead of AC it is AA), interrupted (ACACGTGACACAC small sequence with in repeated sequence) and composite tandem repeats (ACACACAGTGTGTGT two adjacent distinctive sequences) Traditional molecular markers provides insufficient statistical power and accuracy for

Received: January 6, 2019

Reviewed: March 4, 2019

Accepted: March 8, 2019 
estimating genetic differences but the discovery of highly variable loci microsatellites provided more statistical power for deriving the differentiation between groups at risk of endangered species along with the ecological, political and economic aspects of biodiversity protection[2]. These are broadly used as genetic markers, they have particular characteristic in that they exhibits high rates of mutation than the rest of genome. These are widely distributed in genomes have been used extensively for genetic studies such as diversity evolution [3, 4, 5], species identification[6, 7] and gene mapping $[8,9]$ they have been associated with complex diseases. These are the witness to demonstrate the functional impact on genes; hence these are candidates for disease susceptibility alleles. The most important among these are the triplet repeat, more than five triplet repeat expansion causes a range of diseases including Fragile X, Friedreich'sataxix, Mytonic Dystrophy, Huntington's disease and Friedreich's ataxia, several Spinocerebellar ataxias [10]. In diseased individual the repeat copy numbers are more than the normal ranging from tens to hundreds or thousands. Different alleles of this variable number of tandem repeats (VNTR) are identified in type I diabetes [11]. In [12] type II diabetes developed with obese individuals. For example, a tandem repeated GGGCGG polymorphism within the promoter of the 5-Lipoxygenase gene (ALOX5), the first enzyme in the leukotriene biosynthetic pathway, has been shown to play an important role in response to leukotriene modifier therapy. Most individuals carry five repeats of the GGGCGG motif; however, 5 to 35\% of white-skinned or European origin individuals carry at least one allele with three or two repeats at this locus [13]; these tandem repeats are used in target drug identification and validation [14]. Initially the tandem repeats were designed for research in humans, now these will become a powerful tool for animal and plant research, these provides relevant information for identifying conservation units for investigating genetic process. Nature frequently deletes or inserts entries substrings as a unit. An important feature of tandemly repeated DNA is the capacity to vary in terms of length, based on the number of repeat units, this is a characteristic feature of genetic diversity [15]. The study of this class of genetic variation has proved extremely important to our understanding of the human genome.

\subsection{NEXT GENERATION SEQUENCING}

The approach of cutting edge DNA sequencing innovation has made a downpour of succession information that should be put away, sorted out, and conveyed [16]. Recently sequencing cost reduced for every 5 to 7 months DNA data will be doubled. At the same time there is need in new tools to process this much of huge data at a higher rate. The European Bioinformatics Institute (EBI), which has a focal archive of succession information called EMBL-bank, has expanded capacity limit from 2.5 petabytes in 2008 to 5 petabytes in 2009 [17]. All existing tools in this field were single processor based and run on traditional setup. We can attack the problem caused by exponential data growth by applying horizontally scalable techniques [18] from current analytics systems to accelerate scientific processing pipelines, these systems can be built with a set of techniques for implementing scientific analyses efficiently using commodity "big data" systems. Most of the existing tools being currently in use have no support of multi node execution, though some of them support multi-threading, parallel execution, the speed might be limited due to heavy inter process communications when the number of cores is high, the system sometimes busy with resource management than the actual problem solving. As whole genome analysis is gaining attention there is need for new tools to be developed and run on most recent technologies like Hadoop MapReduce framework [18].

\subsection{MAPREDUCE FRAMEWORK}

MapReduce is a software framework that allows certain kinds of parallelizable or distributable problems involving large data sets to be solved using a large number of 
commodity low-end servers in a scale out [19] manner. MapReduce is attractive because it abstracts parallel and distributed concepts in such a way that it allows novice programmers to take advantage of cluster computing without needing to be familiar with associated complexities such as data dependency, replication, reliability and mutual exclusion. The challenge in this framework is problems must be expressed in such a way that they can be solved using MapReduce, however MapReduce addresses the challenges of distributed programming by providing an abstraction that isolates the developer from system level details [19].

Recently there are very few tools in the field of bioinformatics developed on opensource implementation of MapReduce framework called Hadoop to run and manage parallel execution. Blast Reduce [20] designed on MapReduce framework which is 250x faster than BLAST [21] which is widely used application for sequence alignment. Halvade [22]is framework developed for sequence analysis for read alignment in Map phase and variant calling in Reduce phase based on best practices of GATK [23] is able to process a 50 -fold coverage whole genome data set in under $3 \mathrm{~h}$, which is a $90 \%$ efficient compared to other methods. Mining repetitive sequences in a MapReduce setting [24] proposes a method for finding various kinds of repeats by scanning the document once and for each substring of length $\mathrm{k}$ stored in Hbase table along with its starting address, from this table again they tried to find repeats of size $2 \mathrm{k}, 4 \mathrm{k}$ and so on, but it does not find repeats of every possible lengths. Repeats identified from initially stored different k-length subsequence in a Hbase table by scanning every row. This approach requires two scans on the data given, our approach scans given file only once and no need to connect other component like Hbase. This approach requires three jobs, first job process raw sequence and stores the intermediate data in Hbase, second job finds various lengths of repetitive sequences and merges adjacent building blocks in each sequence and final job eliminates non repeats. Kareem $\mathrm{S}$ et al in their parallel NGS data analysis pipeline in Hadoop [25] presented performance comparison of NGS pipeline scalability from one to two and one to ten samples being executed in parallel and observed run time increased as 1.05x, 2.24x only, they concluded as Hadoop based pipeline is 30x faster. Genome Analysis Toolkit (GATK) is framework designed on the philosophy of MapReduce, provides a small set of data access patterns required for majority of analysis tools, MapReduce enabled stability in process, CPU and memory efficiency and automatically parallelizes methods those run on both shared memory machines and distributed clusters [26], but this is not included SSR tools in it. Motif finding problem in large DNA data sets [27] designed in Hadoop framework based on word counting principle. There are other Hadoop based biological applications listed in [28], like seqWare Query Engine[29] based on NoSQLHbase supports database operations on huge number of genomes, this paper recommended redesigning existing tools to run on MapReduce framework. Hadoop also used in Cloudburst[30] to accelerate the read mapping process of short read sequencing data to a reference genome for SNP discovery and genotyping. Tools for SSR were not there so far developed by Hadoop to get the advantage of parallel programming and to meet the current demands in field of bioinformatics.

The importance and using of Hadoop MapReduce and Hbase and its associated open source projects community in the field of bioinformatics for both users and developers increasing day by day. Most of the bioinformatics work not only the horizontal scalable by Hadoop and HBase important, but also the integration and analysis of consequence of large, disparate data sources into one data warehouse under Hadoop with few HBase tables [31]. But there are big challenges of big data with the advent of high throughput genome technologies, life scientists are to face massive data sets, encountering challenges in processing, handling of information [32]. Our approach is also trying to speed up analysis to serve who want to use big data. For example, if 50 or 100 genomes are to be analysed and results compared, number of computational steps are involved. Steps can 
run either in sequential or in parallel; Hadoop framework allows analysis as data continuously increasing. Recently there is a demand raised by experts to adapt bioinformatics curricular to big data [33]. Due to the complexity of biomedical big data, there is a need new professional in this field those are capable of analysing, and interpreting big data by addressing the challenges posed by it. Very recently a study on big data analytics in machine learning perspective addresses several issues and challenges by big data problems in the field of bioinformatics [34]. The existing big data architectures do not provide a comprehensive solution for big data analytics. To get solutions in faster way, with fault tolerant, facilitating incremental, distributed and optimized for iterative and complex computations MapReduce framework recommended for current and future scope in this field. It is being used by many researchers in this field for solving problems with Data Ming principles, one of such problem is for mining maximal contiguous frequent patterns in large DNA sequence datasets in more efficient way [35]. There are some surveys regarding MapReduce operations in bioinformatics concluded importance and vast scope of this technology in this field [36]. Our literature survey found that there are no tools for SSR so far developed by Hadoop to get the advantage of parallel programming and to meet the current demands in field of bioinformatics. Our initiation may help in this direction.

In this paper we developed a group of jobs in Hadoop MapReduce to identify tandem repeats. It follows divide and conquer strategy, input file is divided into parts nearly equal size and assigns to different mappers available in cluster. The entire process explained as follows: Section 2 proposed system architecture; Section 3 describes all algorithms; Section 4 experimental setup, results and their discussion; Section 5 conclusion followed by references.

\section{SYSTEM ARCHITECTURE}

Our method to identify tandem repeats is primarily divided into two parts, one part is for mono repeats identification, in second part other repeats like di, tri, tetra, penta, hexa and k-mer(k up to some desired and suitable). Reason behind this separation is designing the task as batch processing which is most suitable for Hadoop framework. We employ the rapid application development (RAD) [37] process model in developing the tool in less duration; there are two reasons using this process model we can see working model in less time, if any small issues not addressed properly we can correct it immediately. Fig 1 shows detailed architecture and which is most suitable for this framework, it consists 3 MapReduce jobs run in sequential fashion. Inside a job all map tasks and reduce tasks works in parallel. The Hadoop implementation working details are discussed in [1719],[38-39]. All the components in Figs 1 are implemented as MapReduce classes. Entire process completed by three jobs, first job generates motifs in its map phase and qualifies some them as tandem repeats in its reduce phase; second job extracts all distinguished motifs of all sizes (1 to 6) bp; the input for this job is output of job1; third job consolidates all tandem repeats based on the output produced by job 2 . The details are presented in the following algorithms numbered from 1 to 7. 


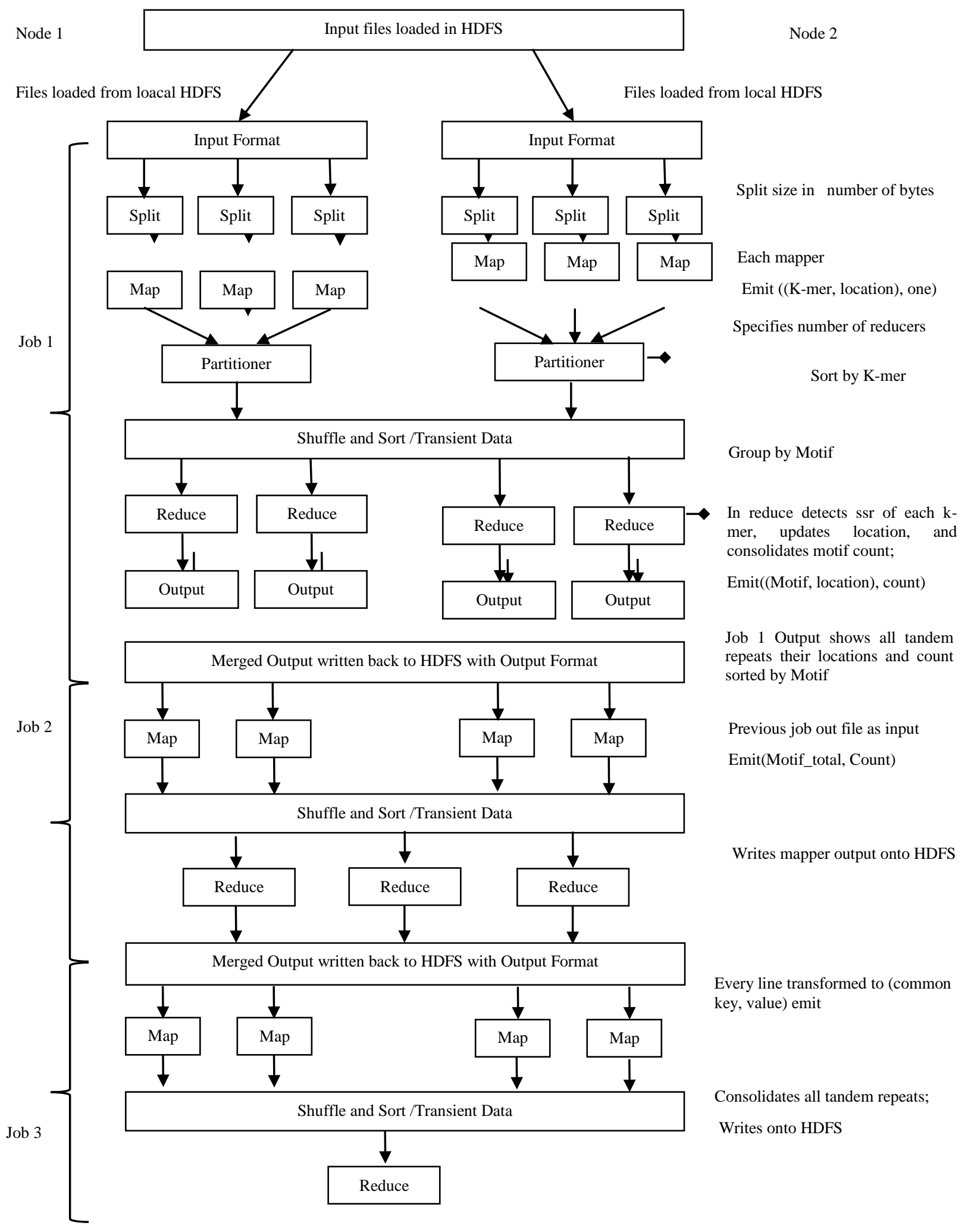

Fig. 1 Work Flow Model for Tandem Repeats by Map Reduce 


\section{PROCESS / IMPLEMENTATION OF FRAMEWORK CUSTOMISED INPUT SPLIT}

As shown in Fig 1 the input data is divided according user defined splits size by using record reader object. One input split is a chunk of the input that is processed by a single map task [38]. Each map processes a single split as a key-value pair. For some applications like word count uses default split in which every line of input file taken a key -value pair, but such a split does not fit to our problem so customized split with number of bytes as size of split will specify number of mappers required to process whole input; every input split has partition number as key. This input splits can be processed in parallel by different machines.

\subsection{MAP PHASE MOTIF GENERATION}

In a map task first pre-process the value with respect to new line character. It extract a $\mathrm{k}-\operatorname{mer}(\mathrm{k}=2$ to 6 in this paper) as motif and emits it with start index, end index, as key, 1 as value. Algorithm 1 shows details of motif generation, map task generates customised key as shown Fig 2.
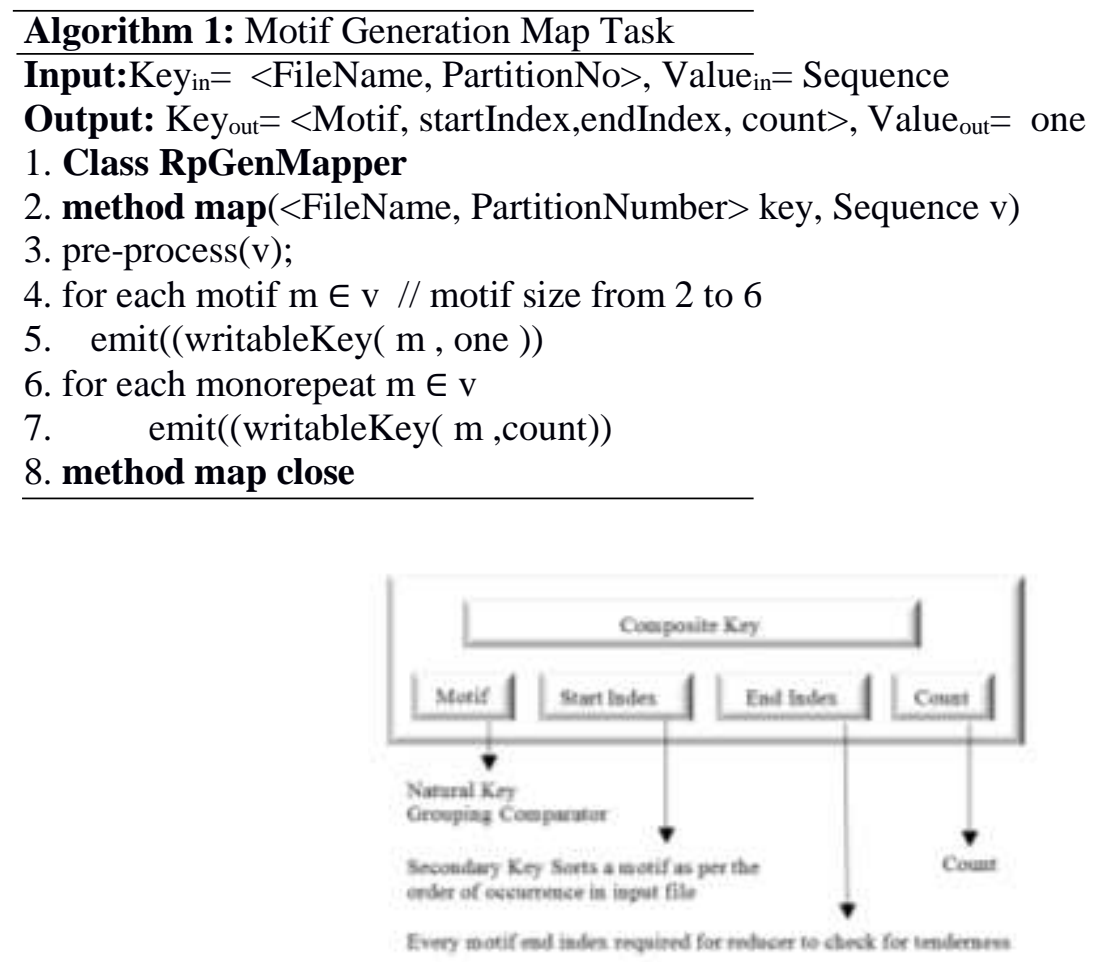

Fig. 2 Composite Key for Partitioning and Sorting

Illustration of map task show in Fig 3. Sends all substrings of length $k ; i, j$ as start, end indices of substring; count 1 . If $\mathrm{k}=3$, assume $\mathrm{G}$ starts at 20 in given file it sends (GAC, 20, 22, 1), (ACT, 21, 23, 1), (CTA, 22, 24, 1) and so on to HDFS [17-19], [38].

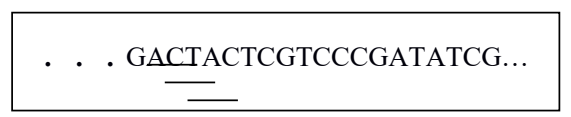

Fig. 3 Extracting a k-mer

The output of every map task given to partitioner [17] which is responsible for dividing 
up the intermediate key space and assigning it to reducers. In other words the partitioner specifies to which reducer intermediate key-value pair must be copied, number of reducers required. The details of partitioner in this job presented in Algorithm 2.

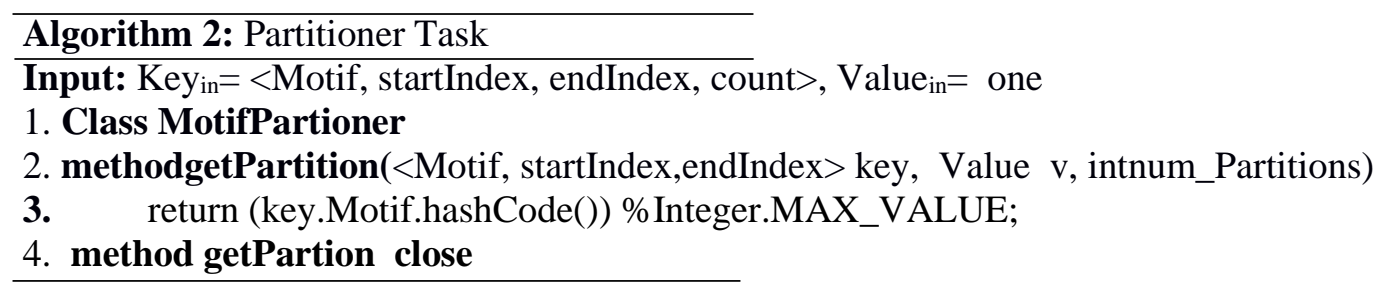

\subsection{REDUCE PHASE TANDEM REPEAT DETECTION}

After successful completion of partition the reduce phase starts each reduce task receives a motif of same kind generated by all mappers in sorted order based on start index of motif. Algorithm 3 presents reduce task in detail.

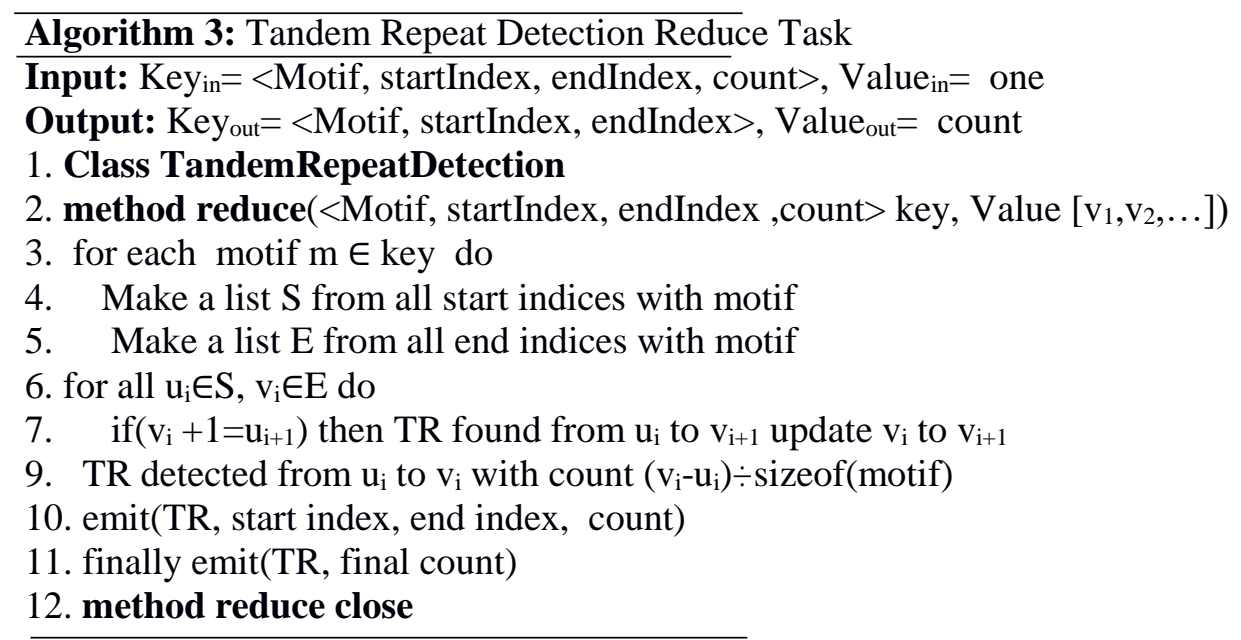

The reduce task compares first motif end index start index of next motif if they are differ by one then that motif is qualified as tandem repeat and emits onto HDFS, all other motifs are simply discarded; Fig 4 illustrates reduce task process.

At the end of first iteration of MapReduce it produces one output file containing all qualified tandem repeats present in the given DNA sequence file. The frame work guarantees intermediate sequences processed in alphabetical order with in a partition. This generates a sorted tandem repeats file in a most convenient form to the biologist to figure out various interested/uninterested motifs also, this also facilitates the biologist search more easily since all tandem repeats were listed in sorted order by considering motif and its start index as key, first sort motif string alphabetically on duplicate motifs sorting takes place on their start indices; at end of particular motif the total count of that motif across whole file is also written on output file. The output file itself convinces the biologist the distribution of motifs, a simple walk on the output file will give a clue on distribution of motifs; by considering a motif, all occurrences of its type are listed one by one with structure shown in Fig 5. Now we can simply compare any two adjacent motifs starting indices, a normalized value from all differences within a motif will give simple distribution of that motif in the given DNA file as shown in Fig 5. 


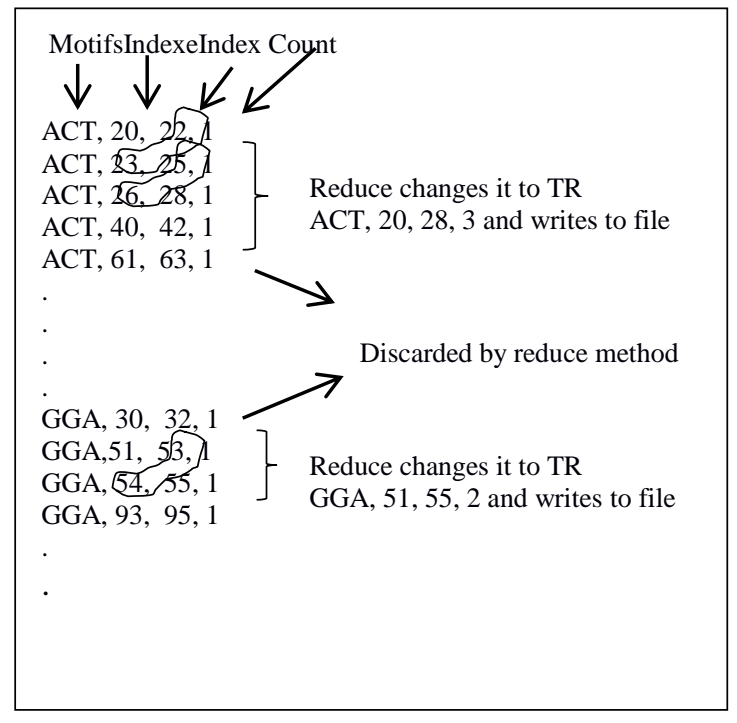

Fig. 4 Verifying tandemness of motif

At the end of first iteration of MapReduce it produces one output file containing all qualified tandem repeats present in the given DNA sequence file. The frame work guarantees intermediate sequences processed in alphabetical order with in a partition. This generates a sorted tandem repeats file in a most convenient form to the biologist to figure out various interested/uninterested motifs also, this also facilitates the biologist search more easily since all tandem repeats were listed in sorted order by considering motif and its start index as key, first sort motif string alphabetically on duplicate motifs sorting takes place on their start indices; at end of particular motif the total count of that motif across whole file is also written on output file. The output file itself convinces the biologist the distribution of motifs, a simple walk on the output file will give a clue on distribution of motifs; by considering a motif, all occurrences of its type are listed one by one with structure shown in Fig 5. Now we can simply compare any two adjacent motifs starting indices, a normalized value from all differences within a motif will give simple distribution of that motif in the given DNA file as shown in Fig 5.

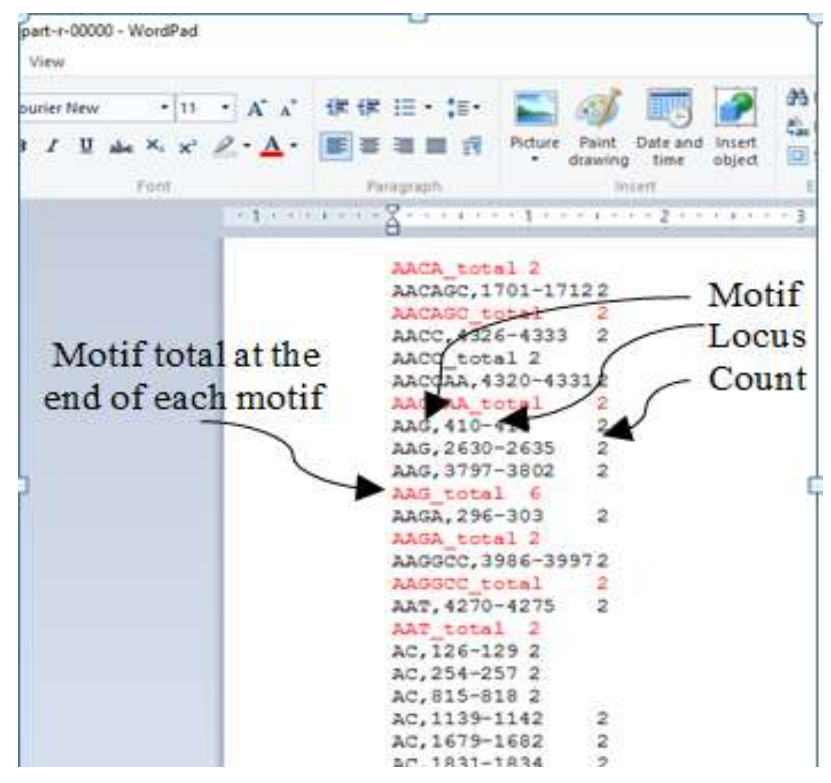

Fig. 5 Output of Job1 


\subsection{EXTRACTION DISTINCT TANDEM REPEATS}

Job 2 extracts all distinguished motifs of all sizes (1 to 6) bp; the input for this job is output of job1. The process is very simple and easy for MapReduce because it is like simply word count program. Every input line segment for job 2 is independent and hence no pre-processing and user defined input splits not required; time required for this, is very less compared to Job1. Process of Job 2 defined in Algorithms 4, 5.

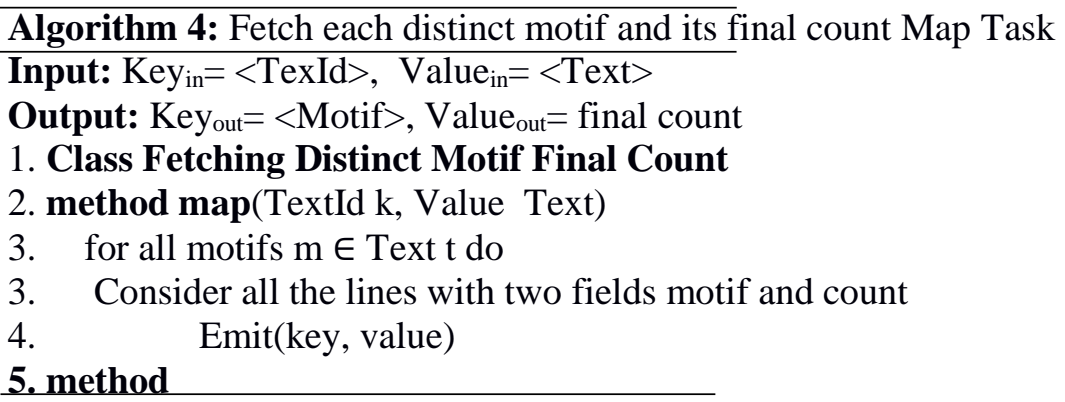

Algorithm5: Reduce Task - simply writes distinct tandem repeats on to output file Input: $K_{\text {ey }}=\langle$ Motifkey $\rangle$, Value in $=\langle$ value $\rangle$

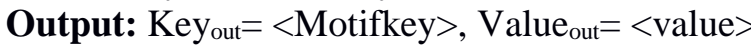

1. Class SimpleTandemRepeatWrites

2. method reduce $(k e y$, value)

3. Emit(key k, value )

4. method reduce close

In map task it fetches all lines with two fields motif total and count shown in Fig 5 and sends to reduce process remaining lines were discarded by map task; the reduce task simply throws these distinguished tandem repeats on to output disk. At the end of Job 2 we have all kinds of tandem repeats of sizes 1 to $6 \mathrm{bp}$ and their count. This output file also in alphabetical order for biologist it is very easy for probe operation, see Fig 6 output segments of job2 "_total" word is appended by job1 to distinguish motif and its total lines, this also helps in map task only considers all words with "_total".

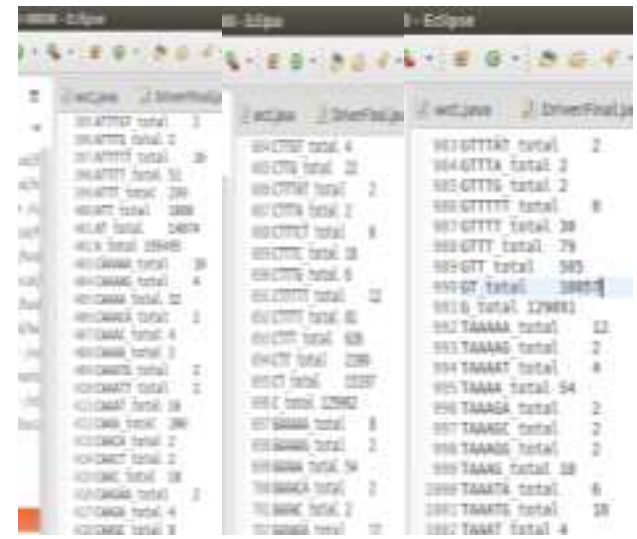

Fig. 6 Output of Job 2

\subsection{CONSOLIDATION TANDEM REPEATS}

Finally, to consolidate all tandem repeats produced by Job 2 another Job 3 started which is very simple like Job 2; we designed Job 3 with map and reduce tasks given in Algorithms 6, 7. The map task accepts line with motif_total, count as Text and sets 
motif_total as a common key, count as value and sends to reducer. Final one reduce task consolidates all values since we changed every key in map task to a common key and hence all are arrived at one reducer, reduce task sums all values see Algorithm 7 and writes single value onto file common key and value. The value is total number of tandem repeats present in given file.
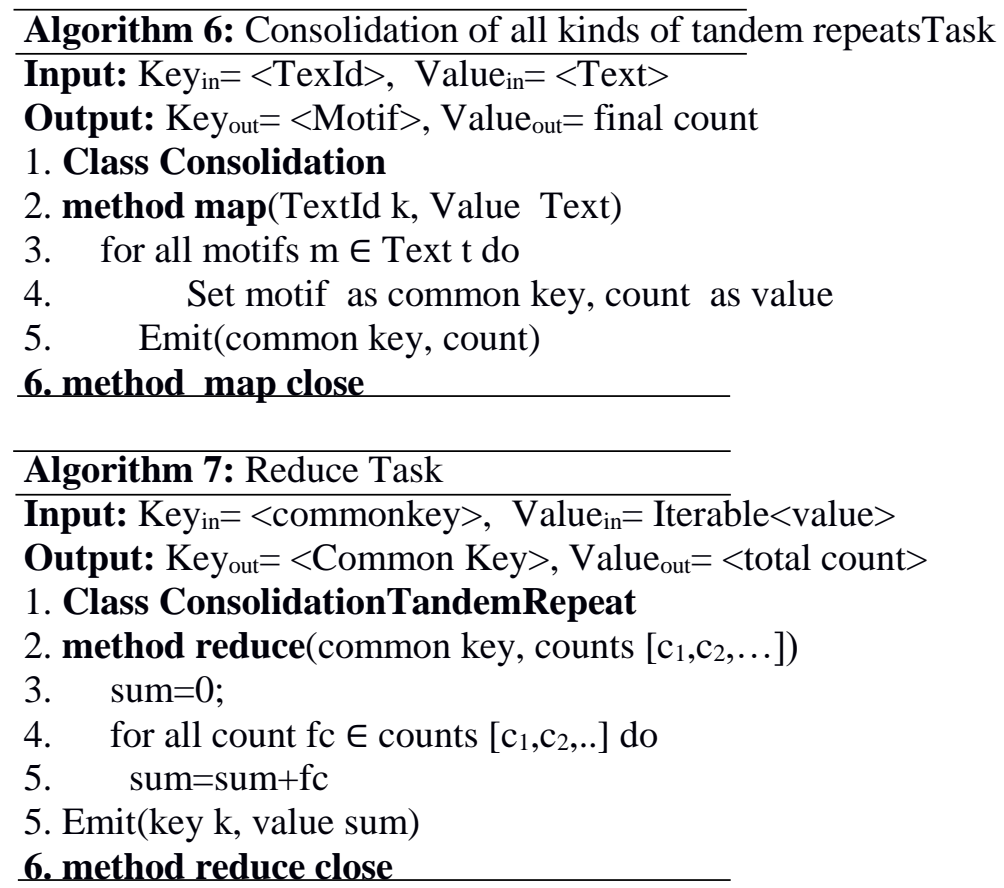

\section{EXPERIMENTAL RESULTS}

We performed our experiments on (1+8 nodes) cluster machine having front node with $2 \mathrm{X}$ Intel ${ }^{\circledR}$ xeon ${ }^{\circledR} \mathrm{E} 5-2640(2.5 \mathrm{GHz} / 6$-core/15MB/95w) processor, $64 \mathrm{~GB}$ RAM, $3 \mathrm{X}$ 600GB HDD and remaining 8 nodes with $1 \mathrm{X}$ Intel® xeon ${ }^{\circledR} \mathrm{E} 5-2640(2.5 \mathrm{GHz} / 6-$ core/15MB/95w) processor, 16GB RAM, 2 X 300GB HDD. The sequences were downloaded from the GenBank sequences are NT_023678(Homo sapiens chromosomes 8 genomic contig), NT_026446 (Homo sapiens chromosome 15 genomic contig)and submitted to tool where it detected large number of tandem repeats compared to tool [40] there is a huge number of difference in repeats approximately 8 to 10 times more. But we are not comparing them directly because of current tool running on a cluster and capable of doing exhaustive computing. Fig 7 shows number of repeats produced by current tool with other tools IMEx [41], TRF [42] our tools produced more number without any assumed computational constraints. 


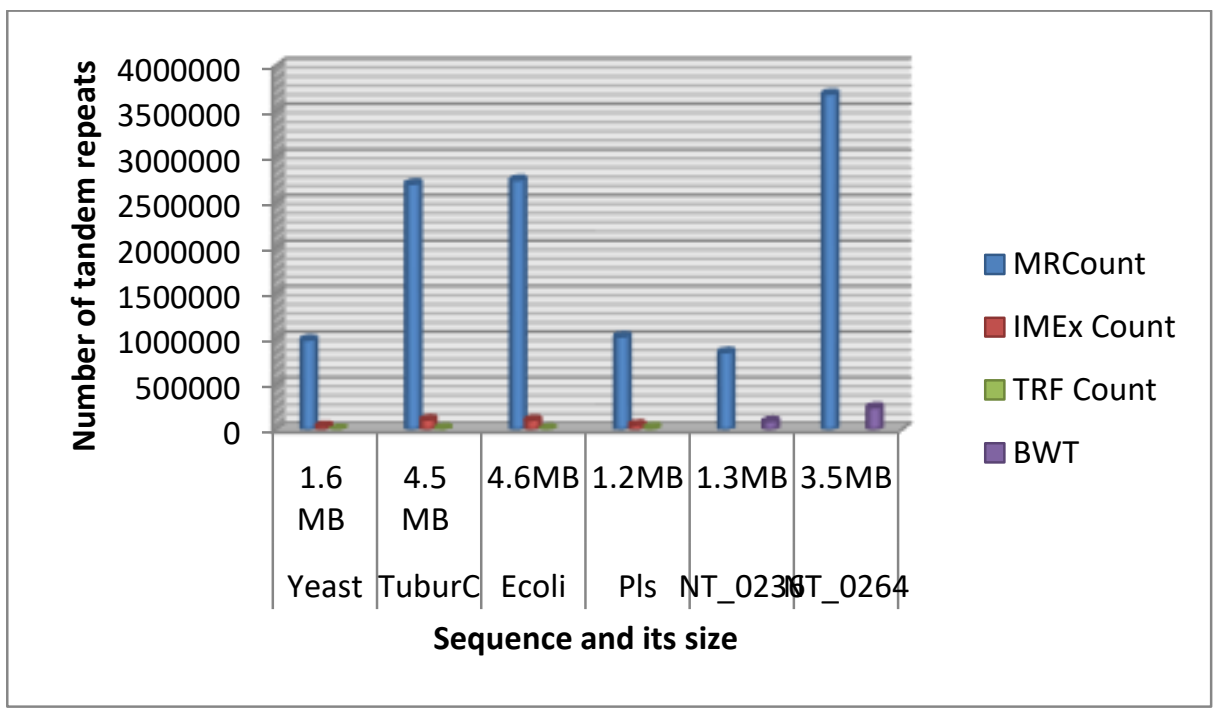

Fig. 7 Number of Tandem Repeats produced by different tools

\section{CONCLUSION}

In this work, authors proposed an efficient and constraint-free Hadoop MapReduce based approach for detecting tandem repeats. Proposed model works in parallel and leverages the functional advantages of key -value based implantations. Experimental results show that the proposed approach more efficient with respect to increased data size when other tools have limitations. Tools for SSR were not there so far developed by Hadoop to get the advantage of parallel programming and to meet the current demands in field of bioinformatics. This framework works in more natural way and exhaustive approach adapted; all existing tools works under some assumed constraints, hence there is a loss in valuable information.

\section{REFERENCES}

[1] Tóth, Gábor, ZoltánGáspári, and Jerzy Jurka. "Microsatellites in different eukaryotic genomes: survey and analysis." Genome research 10.7 (2000): 967-981.

[2] Oliveira, Eder Jorge, et al. "Origin, evolution and genome distribution of microsatellites." Genetics and Molecular Biology 29.2 (2006): 294-307.

[3] J. Tian, T. Isemura, A. Kaga, D.A. Vaughan, N. Tomooka, Genetic diversity of the rice bean (Vignaumbellata) genepool as assessed by SSR markers, Genome 56 (2013) 717-727.

[4] B.W. Legesse, A.A. Myburg, K.V. Pixley, A.M. Botha, Genetic diversity of African maize inbred lines revealed by SSR markers, Hereditas 144 (2007) 10-17.

[5] L.X. Wang, R.X. Guan, Z.X. Liu, R.Z. Chang, L.J. Qiu, Genetic diversity of Chinese cultivated soybean revealed by SSR markers, Crop Sci. 46 (2006) 1032-1038.

[6] D.J. Perry, Identification of Canadian durum wheat varieties using a single PCR, Theor. Appl. Genet. 109 (2004) 55-61.

[7] F. Martin, An application of kernel methods to variety identification based on SSR markers genetic fingerprinting, BMC Bioinf. 12 (2011) 177.

[8] S.K. Biradar, R.M. Sundaram, T. Thirumurugan, J.S. Bentur, S. Amudhan, V.V. Shenoy, B. Mishra, J. Bennett, N.P. Sarma, Identification of flanking SSR markers for a major rice gall midge resistance gene Gm1 and their validation, Theor. Appl. Genet. 109 (2004) 1468-4173.

[9] Y.H. Wang, D.D. Poudel, K.H. Hasenstein, Identification of SSR markers associated with saccharification yield using pool-based genome-wide association mapping in sorghum, Genome 54 (2011) 883-889.

[10] Usdin, K., and E. Grabczyk. 2000. DNA repeat expansions and human disease. Cell Mol Life Sci 57:914-31.

[11] Lucassen, A. M., C. Julier, J. P. Beressi, C. Boitard, P. Froguel, M. Lathrop, and J. I. Bell. 1993. Susceptibility to insulin dependent diabetes mellitus maps to a $4.1 \mathrm{~kb}$ segment of DNA spanning the insulin gene and associated VNTR. Nat Genet 4:305-10. 
[12] Le Stunff, C., D. Fallin, N. J. Schork, and P. Bougneres. 2000. The insulin gene VNTR is associated with fasting insulin levels and development of juvenile obesity. Nat Genet 26:444-6.

[13] Silverman, E. K. H. In, C. Yandava, and J. M. Drazen. 1998. Pharmacogenetics of the 5-lipoxygenase pathway in asthma. ClinExp Allergy 28(Suppl. 5):164-70.

[14] Barnes, Michael R. "4 The Impact of Genetic Variation on DrugDiscovery and Development." In Silico Technologies in Drug Target Identification and Validation (2006): 89.

[15] Julian C. Knight-Human Genetic Diversity_ Functional Consequences for Health and Disease (2009)(1)[chapter 7].

[16] Elaine R. Mardis. The impact of next-generation sequencing technology on genetics. Trends in Genetics, 24(3):133-141, 2008.

[17] Book by Chris Dyer and Jimmy Lin Data-Intensive Text Processing with MapReduce.

[18] J. Dean and S. Ghemawat. MapReduce: simplified data processing on large clusters. Communications of the ACM, 51(1):107-113, 2008.

[19] Lin, Jimmy, and Chris Dyer. "Data-intensive text processing with MapReduce." Synthesis Lectures on Human Language Technologies 3.1 (2010): 1-177.

[20] Schatz, Michael C. "BlastReduce: high performance short read mapping with MapReduce." University of Maryland, http://cgis.cs. umd. edu/Grad/scholarlypapers/papers/MichaelSchatz. Pdf (2008).

[21] Altschul, S.F., et al., Basic local alignment search tool. J MolBiol, 1990. 215(3): p. 403-10.

[22] Decap, Dries, et al. "Halvade: scalable sequence analysis with MapReduce." Bioinformatics 31.15 (2015): 2482-2488.

[23] DePristo, Mark A., et al. "A framework for variation discovery and genotyping using next-generation DNA sequencing data." Nature genetics 43.5 (2011): 491-498.

[24] Cao, Hongfei, et al. "MRSMRS: Mining repetitive sequences in a MapReduce setting." Bioinformatics and Biomedicine (BIBM), 2014 IEEE International Conference on. IEEE, 2014.

[25] Aggour, Kareem S., et al. "A highly parallel next-generation DNA sequencing data analysis pipeline in Hadoop." Bioinformatics and Biomedicine (BIBM), 2015 IEEE International Conference on. IEEE, 2015.

[26] McKenna, Aaron, et al. "The Genome Analysis Toolkit: a MapReduce framework for analyzing nextgeneration DNA sequencing data." Genome research 20.9 (2010): 1297-1303.

[27] Qiang Yu, HongweiHuo, "An Efficient Algorithm for Discovering Motifs in Large DNA Data Sets." IEEE Transactions on NanoBioscience (Volume:14, Issue: 5 ) Page(s):

[28] Xubin, Li, et al. "Hadoop applications in bioinformatics." Open Cirrus Summit (OCS), 2012 Seventh. IEEE, 2012.

[29] B. D. O'Connor, et al., "SeqWare Query Engine: storing and searching sequence data in the cloud," BMC Bioinformatics, vol. 11 Suppl 12, p. S2, 2010.

[30] Schatz M: Cloudburst: highly sensitive read mapping with MapReduce. Bioinformatics 2009, 25(11):1363-1369.

[31] Taylor, Ronald C. "An overview of the Hadoop/MapReduce/HBase framework and its current applications in bioinformatics." BMC bioinformatics 11.Suppl 12 (2010): S1.

[32] Marx, Vivien. "Biology: The big challenges of big data." Nature 498.7453 (2013): 255-260.

[33] Greene, Anna C., et al. "Adapting bioinformatics curricula for big data." Briefings in bioinformatics 17.1 (2016): 43-50.

[34] Kashyap, Hirak, et al. "Big Data Analytics in Bioinformatics: A Machine Learning Perspective." arXiv preprint arXiv:1506.05101 (2015).

[35] Karim, MdRezaul, et al. "A MapReduce framework for mining maximal contiguous frequent patterns in large DNA sequence datasets." IETE Technical Review 29.2 (2012): 162-168.

[36] Zou, Quan, et al. "Survey of MapReduce frame operation in bioinformatics." Briefings in bioinformatics (2013): bbs088

[37] Pressman, Roger S. Software engineering: a practitioner's approach. Palgrave Macmillan, 2005.(Process model).

[38] T. White, Hadoop: The Definitive Guide: O'Reilly Media, Inc.,2009.

[39] Hadoop- Apache Software Foundation project home page.

[40] Pokrzywa, Rafal. "Application of the Burrows-Wheeler Transform for searching for tandem repeats in DNA sequences." International journal of bioinformatics research and applications 5.4 (2009): 432-446.

[41] Mudunuri, Suresh B., and Hampapathalu A. Nagarajaram. "IMEx: imperfect microsatellite extractor." Bioinformatics 23.10 (2007): 1181-1187.

[42] Benson, Gary. "Tandem repeats finder: a program to analyze DNA sequences." Nucleic acids research 27.2 (1999): 573 